International Journal of Engineering Sciences \& Research Technology

(A Peer Reviewed Online Journal)

Impact Factor: 5.164
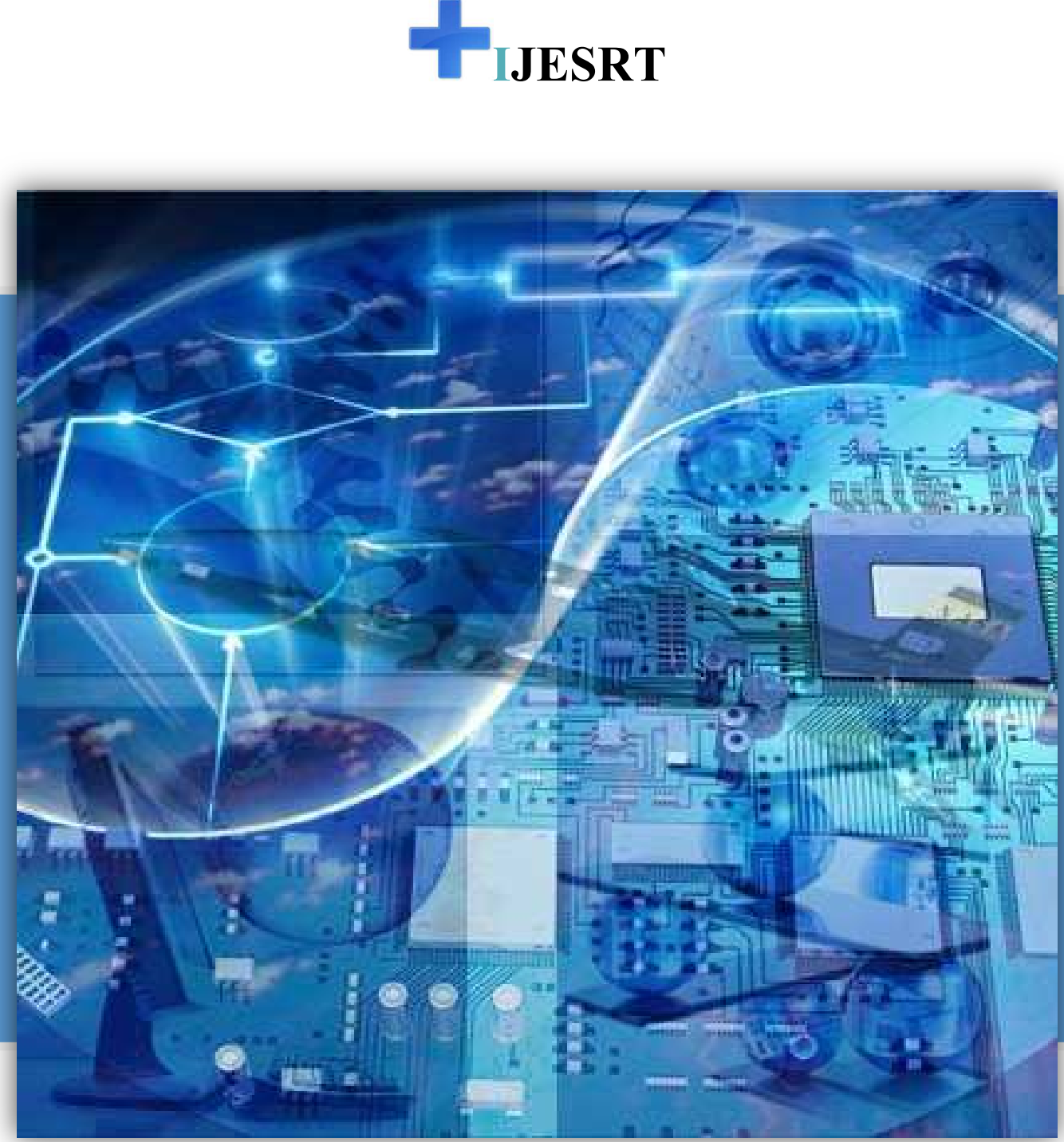

Chief Editor

Dr. J.B. Helonde
Executive Editor Mr. Somil Mayur Shah 
INTERNATIONAL JOURNAL OF ENGINEERING SCIENCES \& RESEARCH TECHNOLOGY

\section{ANALYSIS THE PERFORMANCE AND EFFECTS OF ARTIFICIAL ROUGHNESS WITH VARIOUS RIBS ARRANGEMENT ON THE ABSORBER PLATE OF SOLAR AIR HEATER}

Ashish Ranjan*1, Asst. Prof. V.K.Sinha ${ }^{2}$ \& Prof.M.K.Paswan ${ }^{3}$

${ }^{* 1}$ Research Scholar Jharkhand Rai University Ranchi, Jharkhand, India

${ }^{2}$ Assistant Professor Mechanical Engg. Jharkhand Rai University, Ranchi, Jharkhand, India

${ }^{3}$ professor Mechanical Engg. N.I.T. Jamshedpur, Jharkhand, India

DOI: https://doi.org/10.29121/ijesrt.v9.i11.2020.4

\section{ABSTRACT}

Energy requirement is very important in our life. Today, every country draws its energy needs from a variety of sources. we can broadly categorize these sources as comercial and noncommercial. The commercial sources include the fossil fuels like coal, oil and natural gas, while the noncommercial sources include wood, animal wastes and agricultural wastes. In past few years, it has become obvious that fossil fuel resources are fast depleting and the fossil fuel era is gradually coming to an end. This is particularly true for oil and natural gas. Energy expenditue is constantly increasing all over the world, and we have very little source of energy. So in such a situation, we need to find alternate source of energy. Till this time, it would not be wrong to say that the sun was supplying all the energy needs of man either directly or indirectly. As we know the energy resources available on the earth are in various forms like sunlight, fossil fuels, hydraulic energy, wind energy, tidal energy, geothermal energy and nuclear energy etc. we are having basically two types of energy resources i.e. conventional and nonconventional energy resources. From the point of view of energy saving and fulfill of present and future demand, the best option is renewable energy. We know that Sun is the ultimate source of energy and it is easilly available on the earth in free of cost. Solar energy is used in many areas for different purposes like heat and cool buildings, to heat water, to operate engines etc. So we have best option for provide heat for various purposes is Solar Air Heater. Artificial roughness, which is mounted on the absorber plate, play important role for enhancement of heat transfer rate in order to improve the thermal performance of solar air heater. In this paper our aim is to increase the thermal performance of solar air heater using artificial roughness with different shape of ribs arrangement roughness.

KEYWORDS: Heat transfer, artificial roughness, Thermal performance.

\section{INTRODUCTION}

Solar air heaters are being used for many applications requiring low to medium grade thermal energy, like space heating and cooling, agricultural drying, timber seasoning, mainly due to their low manufacturing cost, simple design, low operating and maintenance cost. Their use limited because of lower thermal efficiencies primarily as a result of lower convective heat transfer coefficient between the absorber plate and air leading to higher plate temperature and greater thermal losses. Various artificial roughness in the form of different geometry like ribs, baffles, wire mesh, dimple shape roughness etc. for the enhancement of heat transfer coefficient and improvement of thermal performance of solar air heaters have been proposed and investigated by a number of investigators. Such roughness geometries includes different rib arrangements like continuous, discrete, transverse, and angled, in v- pattern for ribs of different shapes (circular, square, chamfered, wedge, etc.).[1]. The artificial roughness has been used as an effective means for improvement of thermal performance of solar air heaters. However, this results in increase of friction factor for flowing fluid. In case of v-shape ribs the maximum heat transfer occurred at relative roughness height of 0.034 and at an angle of attack of $60^{\circ}$ [2]. In case of $\mathrm{v}$ - down ribs, two contradictory effects occurs: the secondary flow is towards the central axis where it interacts with the axial flow (at $\mathrm{x}$ in fig.2) creates additional turbulence leadind to the increase in the heat transfer rate [3]. 


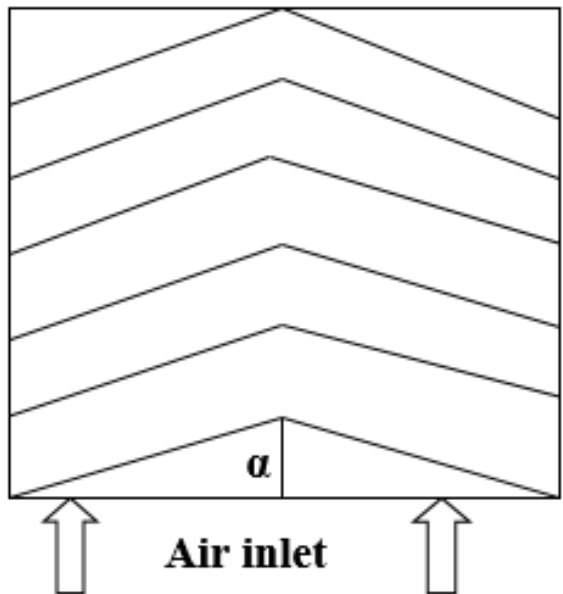

1. V-shape ribs

\section{Primary flow}

\section{$\longrightarrow$ Secondary flow}

$\mathrm{X} \quad$ Mixing of primary and secondary flows

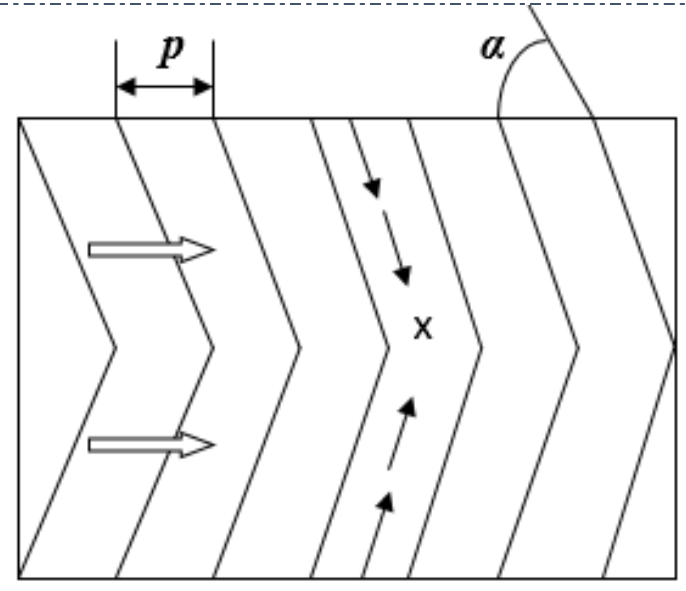

2. V- down continiuos ribs

\section{Nomenclature:}

Collector area, $\mathrm{m}^{2} \mathrm{k}$

Thermal conductivity of air, W/m K

B solar air heater duct height,

$M$, mass flow rate of air, $\mathrm{kg} / \mathrm{s}$

$\mathrm{Cp}$, specific heat of air at constant pressure,

$\mathrm{J} / \mathrm{kg} \mathrm{K}$,Nusselt number

$\mathrm{U}$, hydraulic diameter of solar air heater duct,

$M$, averageNusselt number

$E$,roughness height, $m$ Nusselt number for smooth duct

$\mathrm{E}+$, roughness Reynolds number4, Heat transfer enhancement factor

$\mathrm{E} / \mathrm{U}$, relative roughness height $\mathrm{p}$ pitch of roughness element, $m$

$F$, friction factor $\mathrm{P} / \mathrm{E}$ relative roughness pitch

$F S$, friction factor for smooth duct Re flow Reynolds number

$F r$, friction factor for four sided rough duct Tooutlet temperature of air, ${ }^{\mathrm{C}}$

Average friction factor $T i$ inlet temperature of air, ${ }^{\mathrm{C}}$

Friction enhancement factor average plate temperature, ${ }^{\mathrm{C}} \mathrm{C}$

Friction enhancement factor average air temperature, ${ }^{\mathrm{C}}$

$\mathrm{H}$ convective heat transfer coefficient, $\mathrm{W} / \mathrm{m} 2 \mathrm{~K}$ 


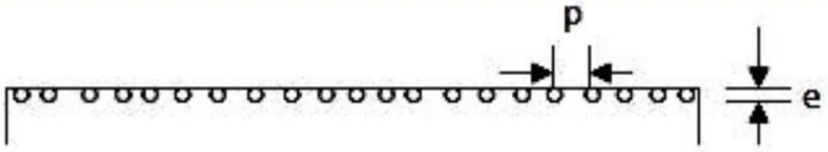

Fluid

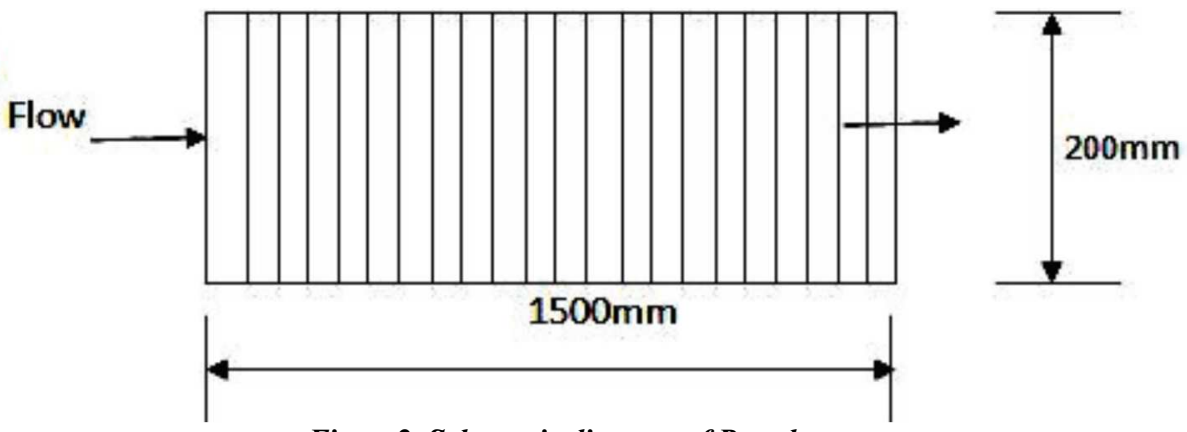

Figure2. Schematic diagram of Roughness

Table 1.Relative roughness pitch (p/e) for a maximum value of a heat transfer coefficient for different types of artificial roughness.

\section{Investigators \\ Abdul-Malik Ebrahimmomin, \\ J.S.Saini,S.C. Solanki (2001) \\ RajendraKarwa, \\ S.C.Solanki,J.S.Saini (2000) \\ J.L.Bhagoria, J.S.Saini,S.C. \\ Solanki (2001) \\ M.K.Paswan et al \\ M.M.Sahu, J.L.Bhagoria \\ (2005) \\ A.R.Jaurker,J.S.Saini (2005) \\ Varun,R.P.Saini,S.K.Singal(2 \\ 007)}

K.R.Aharwal, B.K.Gandhi,

J.S.Saini (2007)

S.V.Krmare, A.N.Tikekar

(2008)

ApurbaLayek, J.S.Saini,S.C.

Solanki (2008)

S.K.Saini,R.P. Saini (2008)

Thakur Sanjay Kumar,Vijay

Mittal, N.S. Thakur,

AnoopGautum (2011)

Sachin Choudhary, Varun,

Manish Kumar Chouhan

(2012)

A.M.Lanjewar, J.L.Bhagoria,

R.M.Sarviya (2012)

Pankaj Kumar \& M.K.Paswan

\section{Roughness Geometry}

$\mathrm{V}$ shaped rib roughness

Integral chamfered rib

Transverse wedge shape

Wire mess Transverse

\&logitudial

$90^{\circ}$ broken transverse rib

Rib-grooved

Combination of inclined \&

transverse ribs

Gap in inclined continuous rib 10

Metal rib grits roughness

Transverse chamfered rib-

groove

Arc shaped wire $\quad 10$

$60^{\circ}$ inclined continuous $\quad 12$

discrete rib

Continuous M shaped ribs

turbulators

W-Shaped

Rhombus shape
10

Value of (p/e) for maximum heat transfer coefficient 10

7.9

7.52

9

20

6

8

htytp: // www.ijesrt.com( $)$ International Journal of Engineering Sciences \& Research Technology [27] 


\section{Investigators}

Abdul-MalikEbrahimmomin, J.S.Saini,S.C. Solanki (2001)

RajendraKarwa,

S.C.Solanki,J.S.Saini (2000)

J.L.Bhagoria, J.S.Saini,S.C.Solanki

(2001)

M.M.Sahu, J.L.Bhagoria (2005)

M.K.Paswan \& S.P .Sharma

A.R.Jaurker,J.S.Saini (2005)

Varun,R.P.Saini,S.K.Singal(2007)

K.R.Aharwal, B.K.Gandhi, J.S.Saini (2007)

S.V.Krmare, A.N.Tikekar (2008)

ApurbaLayek, J.S.Saini,S.C. Solanki (2008)

S.K.Saini,R.P. Saini (2008)

Sanjay Kumar,Vijay Mittal, N.S.

Thakur, AnoopGautum (2011)

Sachin Choudhary, Varun, Manish

Kumar Chouhan (2012)

A.M.Lanjewar, J.L.Bhagoria,

R.M.Sarviya (2012)

Pankaj Kumar \& M.K.Paswan(2016)

\section{Roughness Geometry}

V shaped rib roughness

Integral chamfered rib

Transverse wedge shape

$90^{\circ}$ broken tranvers rib

Wire mess

Rib-grooved

Combination of inclined \& transverse

ribs

Gap in inclined continuous rib

Metal rib grits roughness

Transverse chamfered rib-groove

Arc shaped wire

$60^{\circ}$ inclined continuous discrete rib

Continuous M shaped ribs turbulators

W-Shaped

Rhombus Shape
Value of (e/d) for maximum heat transfer coefficient

0.034

0.041

0.033

0.0338

0.0330

0.0363

0.030

0.0377

0.044

0.03

0.0422

0.0498

0.0777

0.018

0.011

Table 3. Angle of attack ( $\alpha$ ) for a maximum value of a heat transfer coefficient for different types of artificial roughness Investigators

\section{Roughness Geometry}

V shaped rib roughness

Transverse wedge shape

Wire mess

$90^{\circ}$ broken tranverse rib

Gap in inclined continuous rib

Metal rib grits roughness

Transverse chamfered rib-groove

Arc shaped wire

$60^{\circ}$ inclined continuous discrete rib

Continuous $\mathrm{M}$ shaped ribs turbulators

W-Shaped

Rhombus Shape
Value of ( $\alpha$ ) for maximum heat transfer coefficient

$60^{\circ}$

$90^{\circ}$

$90^{0}$

$90^{\circ}$

$60^{\circ}$

$60^{\circ}$

$60^{\circ}$

$(\alpha / 90=0.3333)$

$60^{\circ}$

$60^{\circ}$

$60^{\circ}$

$90^{0}$ 


\section{RESEARCHERID}

THOMSON REUTERS

ISSN: 2277-9655

[Ranjan et al., 9(11): November, 2020]

Impact Factor: 5.164

ICTM Value: 3.00

CODEN: IJESS7

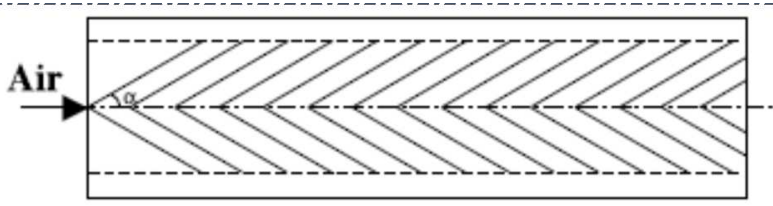

(i)

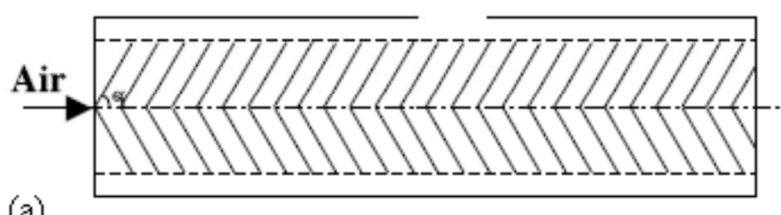

(iii)

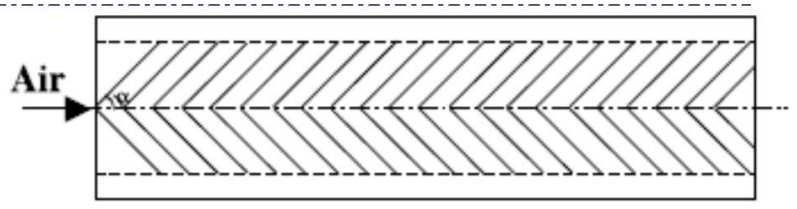

(ii)

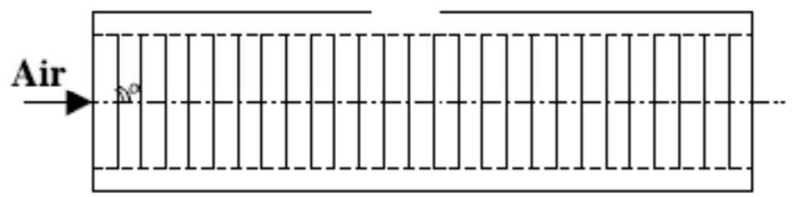

(iv)

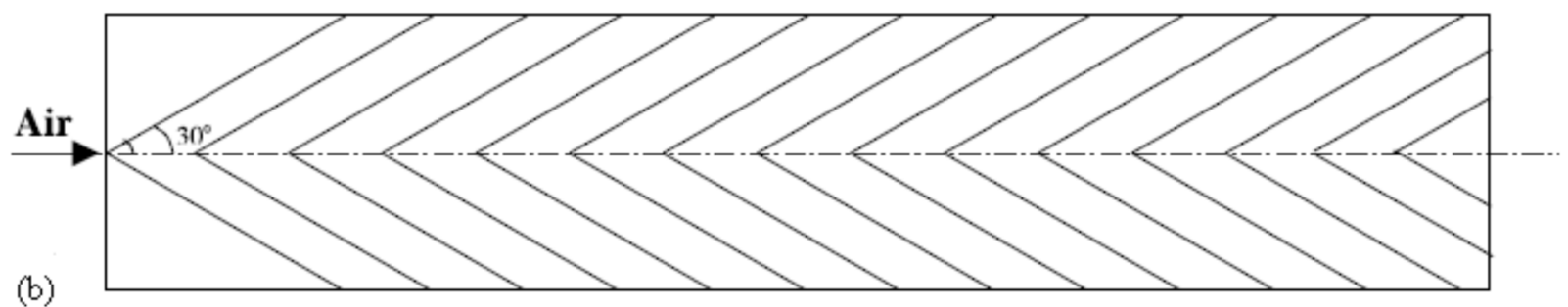

(b)
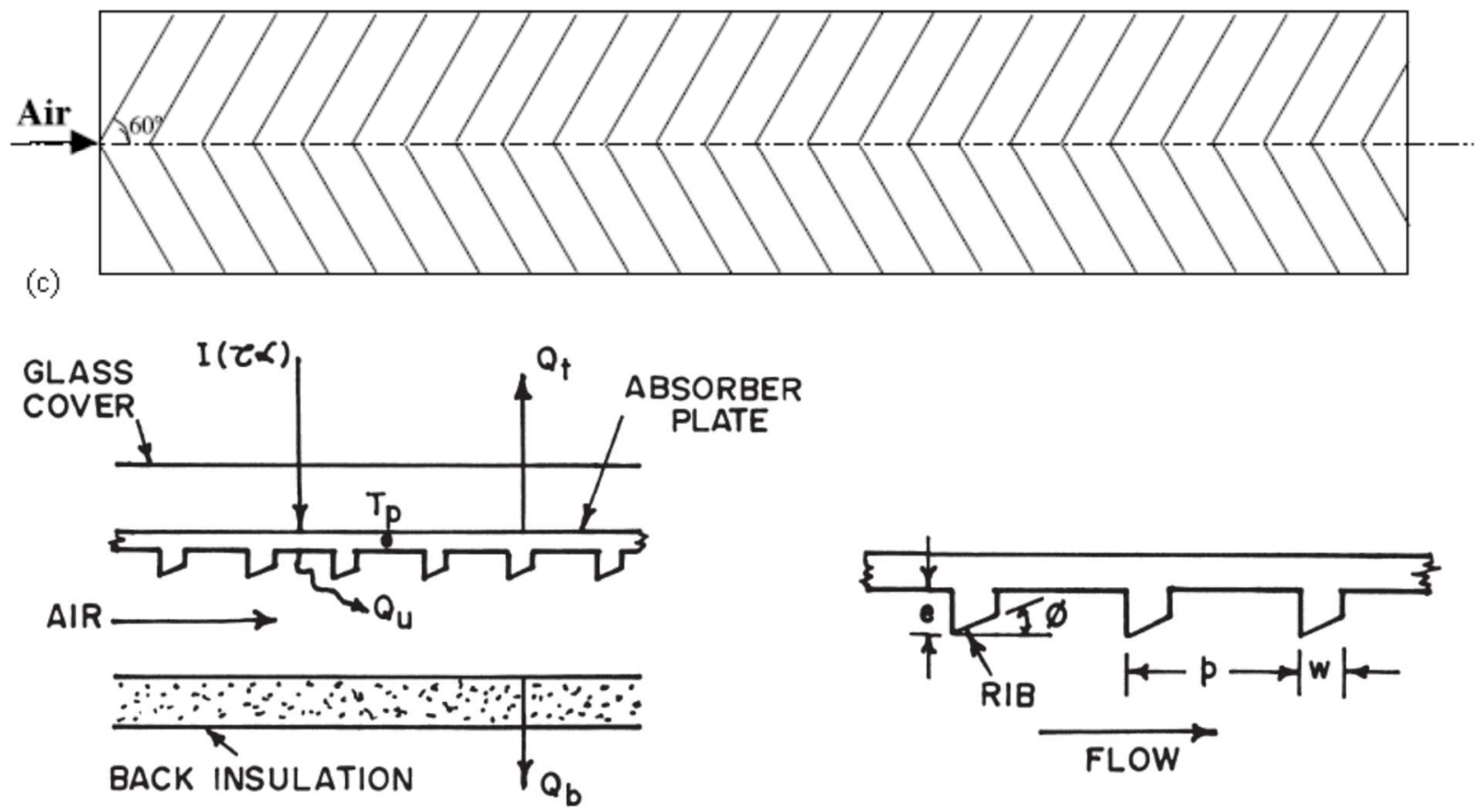

FLOW

htytp: // www.ijesrt.com( $)$ International Journal of Engineering Sciences \& Research Technology [29] 
Table 4. Correlations developed for heat transfer and friction factor for different roughness geometries

Momin,Saini,Solanki

(2001)

Karwa,Solanki,Saini

(2000)

Integral chamfered rib

Wire mesh

(2002)

Bhagoria,Saini,Solanki

(2001)

Varun,Saini,Singal

(2007)

Karmare\&Tikekar (2008) transverse ribs
$\mathrm{V}$-Shaped rib roughness

$\mathrm{Re}-2500-18000$, $(\mathrm{e} / \mathrm{Dh})=0.02-0.034, \alpha=30-$ $90^{\circ}$,pitch-10.

Pitch $-4.58-7.09$, duct depths $21.8,21.5,16 \mathrm{~mm}, \mathrm{Re}$ $-3750-16350$

Pitch- $1.5-6.25$,duct depth$20 \mathrm{~mm}, \mathrm{Re}-3000-20000$

Transverse wedge shape

Combination of inclined \&

Re-2000-14000,Pitch-5$13 \mathrm{~mm}, \mathrm{~W} / \mathrm{H}-10$

Re -3000-18000, roughness height $0.075-0.033 \mathrm{rib}$

Re-3600-17000,Pitch-1517.5, e/Dh- 0.035-0.044
Nur $=$

$0.067 \times(\mathrm{Re}) 0.888 \times(\mathrm{e} / \mathrm{Dh}) 0.4$

$24 \times\left(\alpha / 60^{\circ}\right)-0.077 \times \exp [-$

$\left.0.782 \times\left(\ln \alpha / 60^{\circ}\right) 2\right]$

$\mathrm{Fr}=6.266 \times(\mathrm{Re})-$

$0.425 \times(\mathrm{e} / \mathrm{Dh}) 0.565$

$\times\left(\alpha / 60^{\circ}\right)-0.093 \times \exp [-$

$\left.0.719 \times\left(\ln \alpha / 60^{\circ}\right) 2\right]$

For $7 \leq \mathrm{e}+<20$

$\mathrm{R}=1.66 \mathrm{e}-0.0078 \varphi(\mathrm{W} / \mathrm{H})-$

$0.4(\mathrm{p} / \mathrm{e}) 2.695 \exp [-$

$0.762\{\ln (\mathrm{p} / \mathrm{e})\} 2](\mathrm{e}+)-0.075$

WhenW/H>7.75 use

$\mathrm{W} / \mathrm{H}=7.75$

$\mathrm{g}=103.77 \mathrm{e}-$

$0.006 \varphi(\mathrm{W} / \mathrm{H}) 0.5(\mathrm{p} / \mathrm{e})-$

$2.56 \mathrm{exp}$

$[0.7343\{\ln (\mathrm{p} / \mathrm{e})\} 2](\mathrm{e}+)-0.31$

When $\mathrm{W} / \mathrm{H}>10$ use $\mathrm{W} / \mathrm{H}=10$

For $20 \leq \mathrm{e}+\leq 60$

$\mathrm{R}=1.325 \mathrm{e}-0.0078 \varphi(\mathrm{W} / \mathrm{H})-$

$0.4(\mathrm{p} / \mathrm{e}) 2.695 \exp [-$

$0.762\{\ln (\mathrm{p} / \mathrm{e})\} 2]$

WhenW/H>7.75 use

$\mathrm{W} / \mathrm{H}=7.75$

$\mathrm{g}=32.26 \mathrm{e}-$

$0.006 \varphi(\mathrm{W} / \mathrm{H}) 0.5(\mathrm{p} / \mathrm{e})-$

$2.56 \mathrm{exp}$

$[0.7343\{\ln (\mathrm{p} / \mathrm{e})\} 2](\mathrm{e}+) 0.08$

Nur $=1.89 \times 10$ -

4(Re)1.21(e/Dh)0.426(p/e)2. 94

$[\exp \{-$

$0.71(\ln (\mathrm{p} / \mathrm{e})) 2\}](\varphi / 10)-0.018$ $[\exp \{-1.50(\ln (\varphi / 10)) 2\}]$

$\mathrm{Nu} / \mathrm{Re} 1.213=0.0006 \times(\mathrm{p} / \mathrm{e}) 0$.

0104 and

$\mathrm{Nu}=0.0006 \times \operatorname{Re} 1.213 \times(\mathrm{p} / \mathrm{e}) 0$

.0104

$\mathrm{f} / \mathrm{Re}-$

$0.3685=1.0858 \times(\mathrm{p} / \mathrm{e}) 0.0114$

and

$\mathrm{f}=1.0858 \times \mathrm{Re}-$

$0.3685 \times(\mathrm{p} / \mathrm{e}) 0.0104$

$\mathrm{f}=15.55 \times(\mathrm{Re})-$

$0.26 \times(\mathrm{e} / \mathrm{Dh}) 0.91 \times(1 / \mathrm{s})-0.27$ $\times(\mathrm{p} / \mathrm{e})-0.51$ for $3600<\operatorname{Re}<$ 17000

$\mathrm{Nu}=2.4 \times 0$ -

$3 \times(\mathrm{Re}) 1.3 \times(\mathrm{e} / \mathrm{Dh}) 0.42 \times(\mathrm{l} / \mathrm{s})-$ 0.146 
Saini\&Saini (2008)

Arc shaped wire $\times(\mathrm{p} / \mathrm{e})-0.27$ for $3600<\mathrm{Re}<$ 17000

$\mathrm{Nu}=0.001047 \operatorname{Re} 1.3186(\mathrm{e} / \mathrm{d})$

0.3772

$(\alpha / 90)-0.1198$

$\mathrm{f}=0.14408 \mathrm{Re}-$

$0.17103(\mathrm{e} / \mathrm{d}) 0.1765$

$(\alpha / 90) 0.1185$

Thakur Sanjay Kumar et al. Continuous M shaped ribs turbulators

Re-3000-22000, Pitch-12.5$75, \alpha-30-60^{\circ}, \mathrm{e} / \mathrm{D}-0.037$ 0.0776

$\mathrm{Nu}=3 \times 10-5$

(Re)0.947(e/D)0.290(p/e)5.8 85

$(\mathrm{d} / \mathrm{w}) 0.115 \times \exp [-$

$1.237(\ln (\mathrm{p} / \mathrm{e})) 2]$

$\mathrm{f}=0.014 \mathrm{Re}-$

$0.23(\mathrm{e} / \mathrm{D}) 0.804(\mathrm{~d} / \mathrm{w}) 0.097$

Pankaj Kumar \&

M.K.Paswan (2016)
Rhombus shape
Re-3000-18000,Pitch$18 \mathrm{~mm}$, duct depth-20 mm

\section{CONCLUSION}

- This Paper reviews the investigation carried out by various investigators in order to enhance the heat transfer by use of artificial roughness.

- Use of artificially roughened surfaces with different type of roughness geometries of different shapes,sizes and orientation is found to be the most effective technique to enhance the heat transfer rate with little penalty of friction.

- Roughness in the form of ribs and wire matrix were mainly suggested by different investigators to achieve better thermal performance. Among all, rib roughness was found the best performer as far as thermal performance is concerned.

- Correlations developed for heat transfer and friction factor for solar air heater ducts having artificial roughness of different geometries for different investigators are also shown in tabular form. These correlations can be used to predict the thermal efficiency, effective efficiency and then hydraulic performance of artificial roughened.

\section{REFERENCES}

[1]. Rajeev Ranjan, M.K.Paswan, N.Prasad, CFD Analysis of Thermal Performance in Isosceles Right Triangle Rib Roughness on the Absorber Plate Solar Air Heater, Indian Journal of Science andTechnology,Vol9(38). DOI:10.12485/IJST/2016/V9i38/90171.

[2]. Rajeev Ranjan, M.K.Paswan, N.Prasad and R.V.Sharma Numerical Investigation of Heat transfer and friction factor of Solar Air Heater Provided with Isosceles Right Triangle Rib Roughness on the Absorber Plate, JP Journal of Heat and Mass Transfer. Volume 14, Number 1, 2017, Pages 69-96.

[3]. Momin A-M.E., Saini J.S., Solanki S.C. Heat transfer and friction in solar air heater duct with Vshaped rib roughness on absorber plate. International J. of Heat and Mass Transfer. 2001, 45(16), 3383-3396.

[4]. Karwa R., Solanki S.C., and Saini J.S. Thermo-hydraulic performance of solar air heaters having integral chamfered rib-groove roughness on absorber plates. Energy. 2000, 26,161-176.

[5]. Bhagoria J.L., Saini J.S. and SolankiS.C.,Heat transfer coefficient and friction factor correlations for rectangular solar air heater duct having transverse wedge shaped rib roughness on the absorber plate, Renew.Ene., 25(3), 341-369, (2001).

[6]. Jaurker A.R., Saini J.S., and Gandhi B.K. Heat transfer coefficient and friction characteristics ofrectangular solar air heater duct using rib-grooved artificial roughness. Solar Energy. 2005, 80(8),895907.

[7]. Aharwal K.R, Gandhi B.K, Saini J.S. Heat transfer and friction characteristics of solar air heater duct having gap in integral inclined continuous ribs on absorber plate. International Journal of Heat andMass Transfer. Renewable Energy 33 (2007) 585-596. 
[8]. Layek A., Saini J.S., and Solanki S.C. Heat transfer and friction characteristics for artificially roughened ducts with compound turbulators. International Journal of Heat and Mass Transfer. A. Layek et al. / Renewable Energy 34 (2008) 1292-1298. 